\title{
On the origin of plankton patchiness
}

\author{
J.M.G. Vilar ${ }^{\mathrm{a}}$, R.V. Soléb, J.M. Rubíc, ${ }^{\mathrm{c}, *}$ \\ ${ }^{a}$ Center for Studies in Physics and Biology, The Rockefeller University, 1230 York Avenue, \\ New York 10021, USA \\ ${ }^{\mathrm{b}}$ Department of Physics, FEN, Universitat Politécnica de Catalunya, Campus Nord, Mòdul B4, \\ E-08034 Barcelona, Spain \\ ${ }^{c}$ Departament de Física Fonamental, Facultat de Física, Universitat de Barcelona, Diagonal 647, \\ E-08028 Barcelona, Spain
}

Received 30 April 2002

\begin{abstract}
Plankton is the productive base of aquatic ecosystems and plays a major role in the global control of atmospheric carbon dioxide. Nevertheless, after intensive study, the factors that drive its spatial distribution are still far from being clear. The models proposed so far show very limited agreement with actual data as many of their results are not consistent with field observations. Here, we show that fluctuations and turbulent diffusion in standard prey-predator models are able to accurately and consistently explain plankton field observations at mesoscales $(1-100 \mathrm{~km})$. This includes not only the spatial pattern but also its temporal evolution. We explicitly elucidate the interplay between physical and biological factors, suggesting that the form in which small-scale biotic fluctuations are transferred to larger scales may constitute one of the key elements in determining the spatial distribution of plankton in the sea.
\end{abstract}

(c) 2002 Elsevier Science B.V. All rights reserved.

PACS: 87.23.Cc; 05.40.-a; 87.10.+e; 05.45.-a

Keywords: Complex ecosystems; Plankton patchiness; Prey-predator models; Randomness; Turbulent diffusion; Scales

Understanding how complex ecosystems work often relies on simplified models that disregard many details of the actual system while retaining the essential information [1-3]. In the case of marine ecosystems, not only the simplest approaches failed to explain the spatial distribution of plankton populations but also more sophisticated models were unable to account consistently for the most remarkable features [4]. Even

* Corresponding author. Tel.: +34-93-402-11-62; fax: +34-93-402-11-49.

E-mail address: mrubi@ffn.ub.es (J.M. Rubí). 


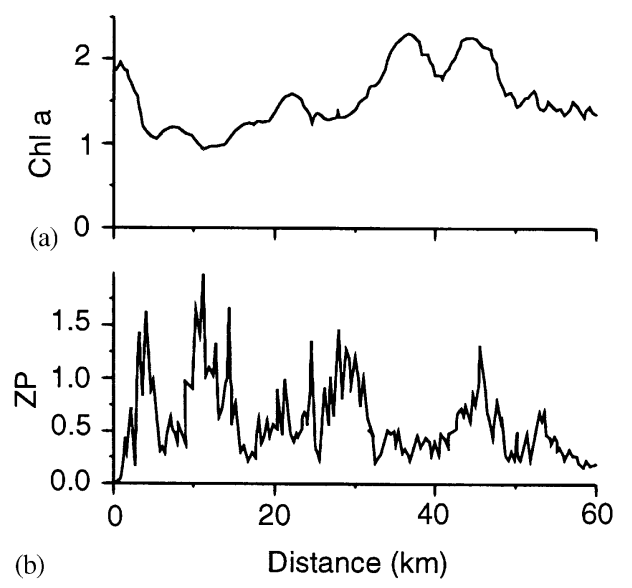

Fig. 1. Transects obtained from field observations for (a) phytoplankton and (b) zooplankton (redrawn from Ref. [5]). Here Chlorophyll $a$ is a measure of phytoplankton activity.

now, it is not clearly understood why under apparent homogeneous conditions, such as temperature and nutrients, plankton is still patchilly distributed. This particular situation is of remarkable importance since in the absence of external sources of patchiness the pattern must arise as a mere consequence of the interactions between the individuals. The typical form of these patterns is illustrated in Fig. 1. The main trait is that zooplankton is more patchilly distributed than phytoplankton $[1,5]$.

The most intuitive model that can be proposed to explain plankton dynamics [2] considers the population densities of prey (phytoplankton), $N$, and predators (zooplankton), $P$ :

$$
\begin{aligned}
& \frac{\partial N}{\partial t}=F_{N}(N, P)+D_{N} \nabla^{2} N, \\
& \frac{\partial P}{\partial t}=F_{P}(N, P)+D_{P} \nabla^{2} P,
\end{aligned}
$$

where $D_{N}$ and $D_{P}$ are diffusion coefficients; and $F_{N}$ and $F_{P}$ are functions that account for the interaction between both species. This class of models is the most frequently used in theories on pattern formation in ecology [2,6,7]. In the case of plankton, they were able to display spatial heterogeneity under homogeneous conditions [8]. Zooplankton, however, was less patchilly distributed than phytoplankton, in contradiction with the observed pattern $[1,8]$.

There are two relevant features that are not taken into account by this kind of models. First, diffusion in the sea is not quantitatively well modeled by usual Fickian diffusion [9]. Both types of diffusion processes will tend to spread and mix the populations, but the specific form in which this is achieved is different. Second, there is always an intrinsic stochasticity associated with the dynamics of the population [10,11]. From birth to death, all processes share some degree of chance. The way randomness manifests in the dynamics of the individuals depends on the scale we are looking at [1]; deterministic 
equations are expected to be valid in the limit of high numbers of individuals [12]. Therefore, a deterministic description may be a reasonable one for phytoplankton alone, but this does not need to be so for zooplankton which has much fewer individuals [13]. More importantly, while phytoplankton interacts mainly with zooplankton, zooplankton interacts also with fish and whales which are far from being evenly distributed.

These two additional features have been incorporated in a prey-predator model:

$$
\begin{aligned}
& \frac{\partial N}{\partial t}=F_{N}(N, P)-\vec{v} \cdot \vec{\nabla} N, \\
& \frac{\partial P}{\partial t}=F_{P}(N, P)-\vec{v} \cdot \vec{\nabla} P+\xi(t),
\end{aligned}
$$

where dispersal is given by advection with a velocity field $\vec{v}[\equiv \vec{v}(\vec{r})]$ that depends on the position $\vec{r}$, and where a noise term $\xi(t)$ has been included.

In general, the effects of the advective terms depend on the precise form of the velocity field. For some turbulent fields [14], the effect of advection can be simplified as follows: given a passive field $f(\vec{r}, t)$ which evolves as

$$
\frac{\partial f}{\partial t}=-\vec{v} \cdot \vec{\nabla} f
$$

the spatial Fourier transform of $f(\vec{r}, t)$ follows from

$$
\frac{\mathrm{d} f_{k}}{\mathrm{~d} t}=-D|k|^{\beta} f_{k},
$$

where $k$ is the wave number and $D$ a constant. In this case, advection can effectively be viewed as a diffusion process with a diffusion coefficient $D_{\text {eff }}(k)=D|k|^{2-\beta}$ that depends on the scale. In contrast to usual Fickian diffusion, the variance of the field is not proportional to $t$ but is given by $\left\langle r^{2}\right\rangle \sim t^{2 / \beta}$. This is the type of time dependence observed for the dispersion of tracers in the sea $[9,15]$, from which one can obtain the explicit value of the parameter $\beta$.

To render our model analytically tractable, we consider the system around a stable state. Fluctuations in zooplankton, $\xi$, move the system away from equilibrium. If the fluctuations are not too large, we can perform a linear expansion of $F_{P}$ and $F_{N}$ :

$$
\begin{aligned}
& F_{N}(N, P)=c_{N}-a_{11} N-a_{12} P, \\
& F_{P}(N, P)=c_{P}+a_{21} N-a_{22} P .
\end{aligned}
$$

Here $c_{N}, c_{P}, a_{11}, a_{12}, a_{21}$, and $a_{22}$ are positive constants. For the simplest form of the noise term [12], Gaussian white and uncorrelated in space $[\langle\xi(\vec{r}, t)\rangle=0$ and $\left.\left\langle\xi(\vec{r}, t) \xi\left(\vec{r}^{\prime}, t^{\prime}\right)\right\rangle=2 \sigma^{2} \delta\left(\vec{r}^{\prime}-\vec{r}\right) \delta\left(t^{\prime}-t\right)\right]$, and for $a_{22} \sim 0$ the variance spectra are given by

$$
S_{N}(k)=\frac{a_{12}^{2} \sigma^{2}}{\left(\tilde{D}_{N}+\tilde{D}_{P}\right) \tilde{D}_{N} \tilde{D}_{P}} \quad \text { and } \quad S_{P}(k)=\frac{\sigma^{2}}{\tilde{D}_{P}} \text {, }
$$

where $\tilde{D}_{N} \equiv D|k|^{\beta}+a_{11}, \tilde{D}_{P} \equiv D|k|^{\beta}+a_{22}$, and $\sigma^{2}$ is the intensity of the noise source. The assumptions involved do not substantially constrain the applicability of the results. 
When $a_{22}$ is not negligible, the expressions become more involved but the qualitative behavior is still the same. In particular, the high wave number limit remains unchanged. On the other hand, the type of noise we have considered is quite general and can arise, among others, from a random distribution of predators feeding on zooplankton or even from the birth process itself [16]. Other types of noise with different properties, e.g. as those induced by turbulence [14,4], are certainly present but we assume that they are not relevant for the spectral properties of the pattern at the mesoscales.

The variance spectra obtained from previous equations display a power-law region with exponent $-3 \beta$ for the phytoplankton and $-\beta$ for the zooplankton. The value of $\beta \sim 0.87$ inferred from diffusion in the sea [15] leads to exponents -2.6 and -0.8 , both in excellent agreement with field data $[1,17,18]$. It is worth emphasizing that the power law behavior appears only for sufficiently high wave number (short scales); for low wave numbers (long scales), the variance spectra is flat, as observed in most field data $[1,17,18]$.

In the same way, one can compute the coherence between two patterns at different times [18], which provides information about the global dynamics. For the phytoplankton this quantity is given by

$$
\frac{N(k, \Delta t)}{N(k, 0)}=\frac{\tilde{D}_{P} \mathrm{e}^{\tilde{D}_{N} \Delta t}-\tilde{D}_{N} \mathrm{e}^{\tilde{D}_{P} \Delta t}}{\tilde{D}_{P}-\tilde{D}_{N}},
$$

where $N(k, \Delta t)=\int_{0}^{\infty}\|N(k, \omega)\|^{2} \mathrm{e}^{-\mathrm{i} \omega \Delta t} \mathrm{~d} \omega$. This result indicates that short scales lose their correlation faster than long ones and that eventually the whole pattern will be decorrelated, as observed in satellite measurements [18].

In Fig. 2 we plot the typical form of the variance spectra and the squared coherence for different time lags. Both of them are in excellent agreement with field data [1,17-19]. Remarkably, the main properties of the pattern already appear in the linear regime. Therefore, non-linear interactions that drive the system towards a stable state will lead to similar results. To study this aspect in more detail, we have performed numerical simulations for typical non-linear interactions as explained in the caption of Fig. 3. The resulting two-dimensional spatial distribution, transects, and variance spectra (shown in Fig. 3) agree with both the linear model and field data. Other types of nonlinear interactions - e.g. different functional responses-as well as different types of noise - e.g. acting on zooplankton growth rate-also produce similar results (data not shown).

Field observations indicate that the power law region of the variance spectra and the value of the exponent of this power law are robust properties of the system; i.e., these properties are present under a wide variety of conditions. In our model, there is always a power law region whose exponent does not depend on biotic factors but is completely determined by the specific form in which turbulent diffusion acts on the system. This provides a straightforward explanation of the predominance of the observed exponents for the phytoplankton falling between -3 and -2 . These are the values that arise for $2 \mathrm{D}(\beta=1)$ and $3 \mathrm{D}(\beta=2 / 3)$ isotropic turbulence, respectively [14]. In the sea, the value of this exponent will depend on the particular situation, but it is reasonable to assume that it will be between those of $2 \mathrm{D}$ and $3 \mathrm{D}$ isotropic 

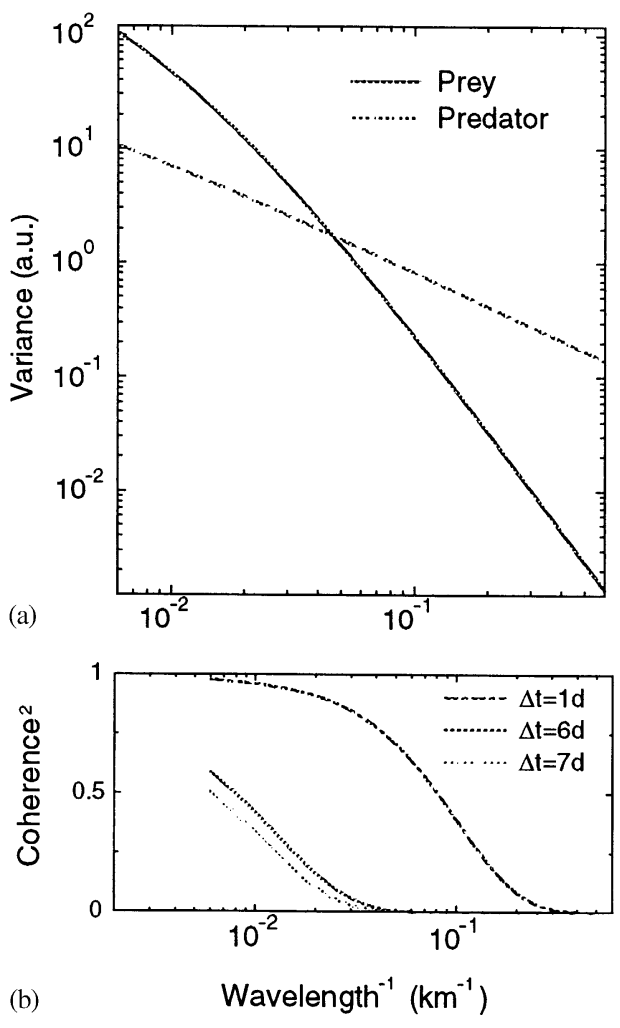

Fig. 2. (a) Variance spectra $S(k)$ for prey and predators from Eqs. (1), (2), and (7). (b) Square coherence $[N(k, \Delta t) / N(k, 0)]^{2}$ for prey (Eq. (9)) for time lags of 1,6 , and 7 days. The values of the parameters are $D=12, a_{11}=0.3, a_{22}=0.05$, and $\beta=1$. The length and time units are $\mathrm{km}$ and days, respectively.

turbulence, as the available data shows $[9,15]$. There are also non-robust properties, such as the region where the variance spectra turns flat. In the model, this depends on many factors: e.g. growth and death rate, and turbulence. Field data shows that, indeed, the position of this region exhibits great variability and that sometimes it is not even present in the range of scales observed.

Turbulent diffusion and noise are two obvious features that have already been considered in the context of marine ecosystems, but none of them by itself has been able to explain the mesoscale patterns. In particular, it is well known that noise generates variability, i.e., that noise can be a source of patchiness [20,21]. For instance, reactiondiffusion prey-predator models with noise produce patterns that at a glance strongly resemble those observed in the sea $[22,23]$. The exponents obtained ( -6 for the phytoplankton and -2 for the zooplankton), however, are far from the observed ones. This quantitative, but not qualitative, disagreement is due to the dependence of the effective diffusion coefficient with the scale. Thus, reaction-diffusion models are unable to integrate correctly the scale dependence of the physical properties of the environment. 


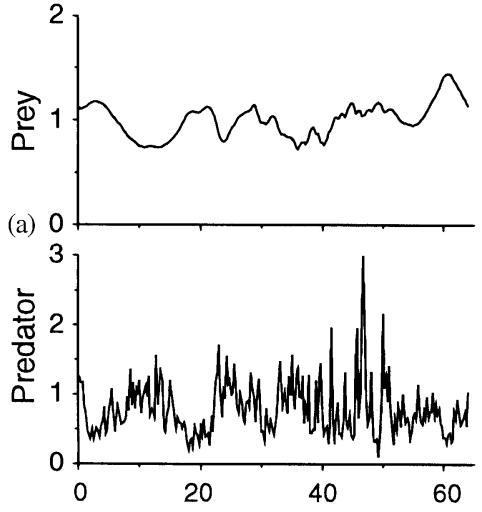

(b)

Distance $(\mathrm{km})$

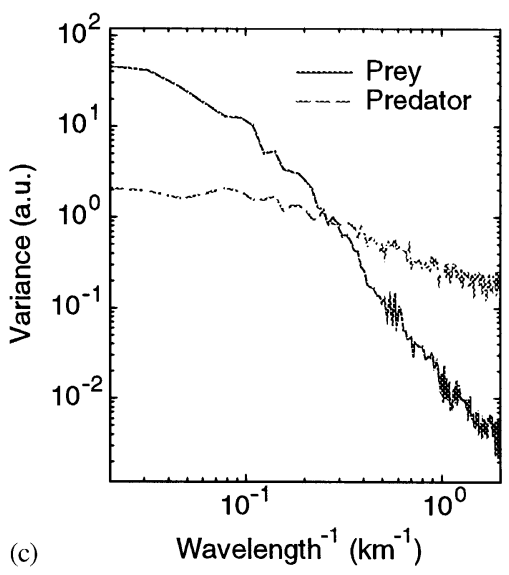

Fig. 3. Numerical simulations for a prey-predator model given by Eqs. (1) and (2), with $F_{N}(N, P) \equiv$ $r N(1-N / K)-c P f(N)$ and $F_{P}(N, P)=P(g f(N)-\varepsilon)$. The functional response is $f(N)=N^{2} /\left(1+N^{2}\right)$. Here, $K, c, g, r$ and $\varepsilon$ are positive constants. The velocity field $\vec{v} \equiv \vec{v}(\vec{r})$ consists of a series of vortices distributed as in Ref. [27] and the noise is assumed to be Gaussian with zero mean and correlation function $\left\langle\xi(\vec{r}, t) \xi\left(\vec{r}^{\prime}, t^{\prime}\right)\right\rangle=2[\sigma P(\vec{r}, t)]^{2} \delta\left(\vec{r}^{\prime}-\vec{r}\right) \delta\left(t^{\prime}-t\right)$. Typical transects for (a) prey and (b) predators. (c) Variance spectra for prey and predators. These results were obtained by discretizing the corresponding equations on a $250 \times 2502 \mathrm{D}$ mesh [28], with periodic boundary conditions and then by using a standard method for integrating stochastic differential equations [29]. The values of the parameters are $r=0.3, K=4, c=2$, $g=0.1, \epsilon=0.05$, and $\sigma=3.5$. The length and time units are $\mathrm{km}$ and days, respectively. The size of each cell of the discretized mesh is $0.25 \times 0.25 \mathrm{Km}^{2}$.

When this is taken into account, noise not only generates patterns but is also able to produce the right ones.

Turbulence plays a somehow ambivalent role. It can act in the same way as diffusion does (transferring variance from smaller to larger scales) and also in the opposite way (from larger to smaller scales). These two types of processes are referred to as turbulent diffusion and turbulent stirring, respectively. The former is the one we have considered in our model. It was already considered in Ref. [4] together with the type of noise that turbulence induces but without the noise that can arise from biotic factors. The latter only plays an important role when some degree of environmental heterogeneity is present [24]. Indeed, it has been shown that turbulent stirring can generate patterns that resemble the observed ones if spatial heterogeneity and time delays are introduced in the model [25]. The type of time delays introduced, however, can lead non-realistic situations such as growing zooplankton in the absence of phytoplankton.

Finally, it is important to emphasize that in our case noise is the key element that allows moving from the individual to the population description. Our results suggest that zooplankton dynamics at lower scales affects the pattern at the mesoscale in the same way as noise does. Considering a more detailed description is not necessary to explain and to understand the main characteristics of the pattern. This does not mean that the actual dynamics of zooplankton is not important at all: its growth rate, its survival, and the intensity of noise itself depend, among other factors, on how 
zooplankton aggregates and on how it avoids its predators [26]. It rather means that under a wide range of conditions all those intricate mechanisms will lead to patterns with properties as those induced by noise.

\section{Acknowledgements}

This work was supported in part by the DGICYT of the Spanish Government.

\section{References}

[1] S.A. Levin, The problem of pattern and scale in ecology, Ecology 73 (1992) 1943-1967.

[2] J.D. Murray, Mathematical Biology, Springer, Berlin, 1988.

[3] J. Bascompte, R.V. Solé (Eds.), Modelling Spatiotemporal Dynamics in Ecology, Springer, Heidelberg, 1997.

[4] T.M. Powell, A. Okubo, Turbulence, diffusion and patchiness in the sea, Phil. Trans. Roy. Soc. London B 343 (1994) 11-18.

[5] D.L. Mackas, Horizontal spatial variability and covariability of marine phytoplankton and zooplankton, Dissertation, Dalhousie University, Halifax, Nova Scotia, Canada, 1977.

[6] R.M. May, Stability and Complexity in Model Ecosystems, Princeton University Press, Princeton, 1974.

[7] H.M. Hassell, H. Comins, R.M. May, Spatial structure and chaos in insect population dynamics, Nature 353 (1991) 255-258.

[8] S.A. Levin, L.A. Segel, Hypothesis for origin of planktonic patchiness, Nature 259 (1976) 659.

[9] A. Okubo, Diffusion and Ecological Problems: Mathematical Models, Springer, Berlin, 1980.

[10] N.S. Goel, S.C. Maitra, E.W. Montroll, On the volterra and other nonlinear models of interacting populations, Rev. Mod. Phys. 43 (1971) 231-276.

[11] R. Durrett, S.A. Levin, Stochastic spatial models: a user's guide to ecological applications, Phil. Trans. Roy. Soc. London B 343 (1994) 329-350.

[12] N.G. van Kampen, Stochastic Processes in Physics and Chemistry, North-Holland, Amsterdam, 1981.

[13] A. Tsuda, H. Sugisaki, T. Ishimaru, T. Saino, T. Sato, White-noise-like distribution of the oceanic copepod Neocalanus cristatus in the subarctic North Pacific, Mar. Ecol. Prog. Ser. 97 (1993) 39-46.

[14] A.J. Majda, P.R. Kramer, Simplified models for turbulent diffusion: theory, numerical modelling, and physical phenomena, Phys. Rep. 314 (1999) 238-574.

[15] A. Okubo, Oceanic diffusion diagrams, Deep-Sea Res. 18 (1971) 789-802.

[16] W.R. Young, A.J. Roberts G. Stuhne, Reproductive pair correlations and the clustering of organisms, Nature 412 (2001) 328-331.

[17] L.H. Weber, S.Z. El-Sayed, I. Hampton, The variance spectra of phytoplankton, krill and water temperature in the Atlantic Ocean south of Australia, Deep-Sea Res. 33 (1986) 1327-1343.

[18] K.L. Denman, M.R. Abbot, Time evolution of surface chlorophyll patterns from cross-spectrum analysis of satellite color images, J. Geophys. Res 93 (1988) 6789-6798.

[19] J.F.R. Gower, K.L. Denman, R.J. Holyer, Phytoplankton patchiness indicates the fluctuation spectrum of mesoscale oceanic structure, Nature 288 (1980) 157-159.

[20] J.H. Steele, Spatial heterogeneity and population stability, Nature 248 (1974) 83.

[21] J.M.G. Vilar, R.V. Solé, Effects of noise in symmetric two-species competition, Phys. Rev. Lett. 80 (1998) 4099-4102.

[22] J.H. Steele, E.W. Henderson, A simple model for plankton patchiness, J. Plankton Res. 14 (1992) $1397-1403$.

[23] P. Ballm, The Self-Made Tapestry: Pattern Formation in Nature, Oxford University Press, Oxford, 1998, p. 231.

[24] H. Tennekes, J.L. Lumley, A First Course in Turbulence, MIT Press, Cambridge, 1972.

[25] E.R. Abraham, The generation of plankton patchiness by turbulent stirring, Nature 391 (1998) $577-580$. 
[26] G. Flierl, D. Grünbaum, S. Levin, D. Olson, From individuals to aggregations: the interplay between behaviour and physics, J. Theoret. Biol. 196 (1999) 397-454.

[27] P.P.G. Dyke, T. Robertson, The simulation of offshore turbulent dispersion using seeded eddies, Appl. Math. Model. 9 (1985) 429-433.

[28] W.H. Press, B.P. Flannery, S.A. Teukolsky, W.T. Vetterling, Numerical Recipes, Cambridge University Press, New York, 1986.

[29] P.E. Kloeden, E. Platen, Numerical Solution of Stochastic Differential Equations, Springer, Berlin, 1995. 